\title{
Radiative hydrodynamic simulations of turbulent convection and pulsations of Kepler target stars
}

\author{
Irina N. Kitiashvili ${ }^{1,2,3}$ \\ ${ }^{1}$ Hansen Experimental Physics Laboratory, Stanford University, Stanford, CA 94305, USA \\ email: irinasun@stanford.edu \\ ${ }^{2}$ Center for Turbulence Research, Stanford University, Stanford, CA 94305, USA \\ ${ }^{3}$ Kazan Federal University, Kazan, 420008, Russia
}

\begin{abstract}
The problem of interaction of stellar pulsations with turbulence and radiation in stellar convective envelopes is central to our understanding of excitation mechanisms, oscillation amplitudes and frequency shifts. Realistic ("ab initio") numerical simulations provide unique insights into the complex physics of pulsation-turbulence-radiation interactions, as well as into the energy transport and dynamics of convection zones, beyond the standard evolutionary theory. $3 \mathrm{D}$ radiative hydrodynamics simulations have been performed for several Kepler target stars, from M- to A-class along the main sequence, using a new 'StellarBox' code, which takes into account all essential physics and includes subgrid scale turbulence modeling. The results reveal dramatic changes in the convection and pulsation properties among stars of different mass. For relatively massive stars with thin convective envelopes, the simulations allow us to investigate the dynamics the whole envelope convection zone including the overshoot region, and also look at the excitation of internal gravity waves. Physical properties of the turbulent convection and pulsations, and the oscillation spectrum for two of these targets are presented and discussed in this paper. In one of these stars, with mass $1.47 M_{\odot}$, we simulate the whole convective zone and investigate the overshoot region at the boundary with the radiative zone.
\end{abstract}

Keywords. stars: Hertzsprung-Russell diagram, oscillations, convection, plasmas, turbulence, methods: numerical

\section{Introduction}

It is known that oscillations of a star contain important information about the interior structure and physical and dynamical properties of turbulent convection. Recent progress in observational capabilities and theory opens new challenges for understanding the dynamical evolution of stars. Identification of high angular degree (high- $l$ ) modes is very tricky because of complicated interactions of turbulent convection, radiation, effects of differential rotation and magnetic field (e.g. Benomar et al. 2012a). The unprecedented quality of observations made by Kepler (Chaplin et al. 2011; Uytterhoeven et al. 2011) allows us to estimate physical and dynamical properties of stars more accurately (e.g. Verner et al. 2011; Benomar et al. 2012b), thus providing additional constraints to theoretical models (e.g. Kjeldsen et al. 2008; Bonaca et al. 2012).

Numerical modeling of the convective interior dynamics can provide important clues on how to connect theoretical models and observations. Recent advances in computational capabilities have allowed modelers to develop 3D models, which revealed significant deviations from previous 2D models (Guzik 2011). In this paper I present initial results of new realistic modeling of stellar convection. 


\section{Realistic modeling of stellar convection}

It is known that helioseismology measurements and surface observations of the solar radius and abundances have discrepancies, which can be related to highly turbulent properties of the near-surface layers (Lefebvre et al. 2006; Guzik \& Mussack 2010). Therefore accurate modeling of subsurface turbulent layers is critically important for our understanding of sources of the observed oscillations, and of properties of stellar convection. The simulations of the stellar convection were performed using a 3D radiative MHD code 'StellarBox'. The code was carefully tested and compared with other similar codes (e.g. Jacoutot et al. 2008b). It has been used for the investigation of oscillatory and dynamical properties of solar magnetoconvection (e.g. Jacoutot et al. 2008a; Kitiashvili et al. 2011a,b), and compared with high-resolution observations (e.g. Kitiashvili et al. 2010, 2013).

We perform realistic hydrodynamic simulations of stellar convection, where initial interior structure models are chosen to be consistent with observed surface properties of stars. The initial background models of the stellar interior structure were obtained using the standard 1D stellar evolution code 'CESAM' (Morel 1997; Morel \& Lebreton 2008). These models of the interior structure were used as initial conditions in our numerical model for selected Kepler target stars with masses ranging from 1.01 to $1.52 M_{\odot}$ (Fig. 1). The stellar masses were approximately estimated from a grid of the calculated stellar models to match observed spectroscopic parameters. The simulations were performed for a small volume of the upper convective zone and the lower atmosphere, confined in a 'box domain', with laterally periodic boundary conditions, and top and bottom boundary conditions open for the radiation energy flux.

Our numerical simulations showed that the dynamics of convective flows in these stars strongly deviate from the solar convection dynamics. Figure 2 shows a horizontal cut of the temperature distribution in the photosphere (panel $a$ ) and a vertical cut of the
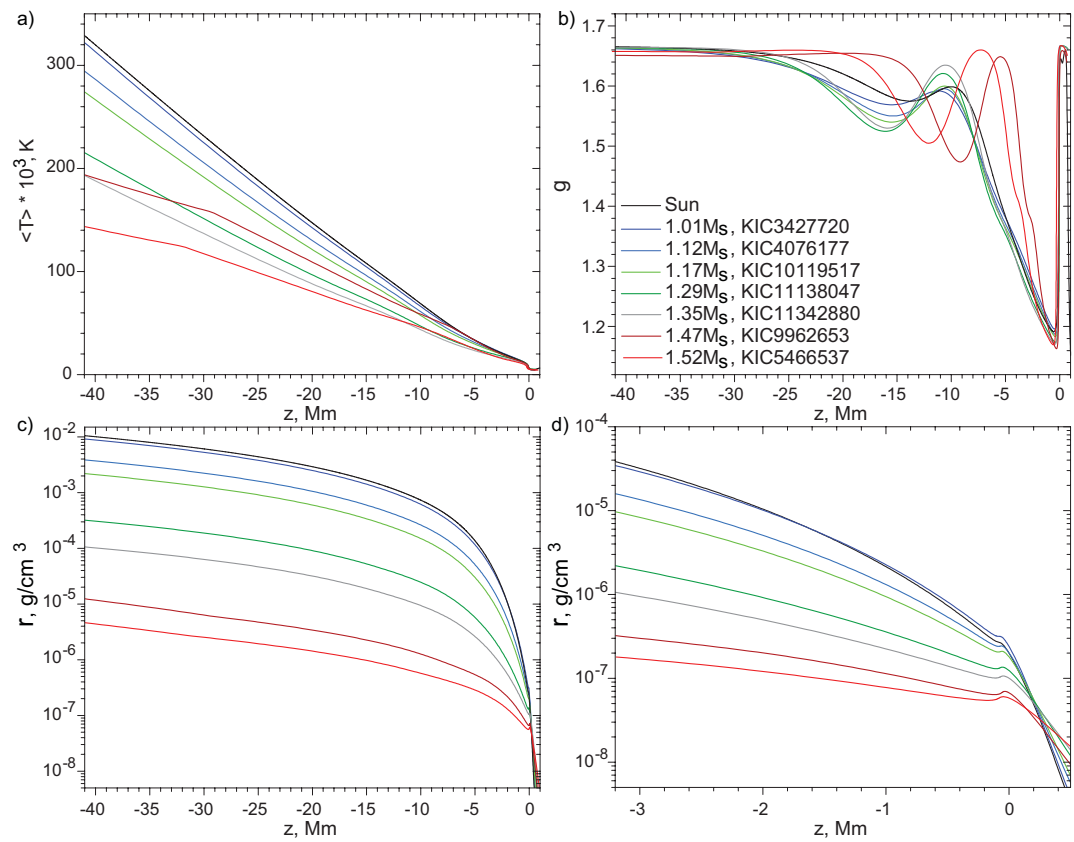

Figure 1. (a) Vertical profiles of temperature, $(b)$ adiabatic exponent, $\gamma$, and $(c-d)$ density for Kepler target stars with masses from 1.01 to $1.52 M_{\odot}$. 

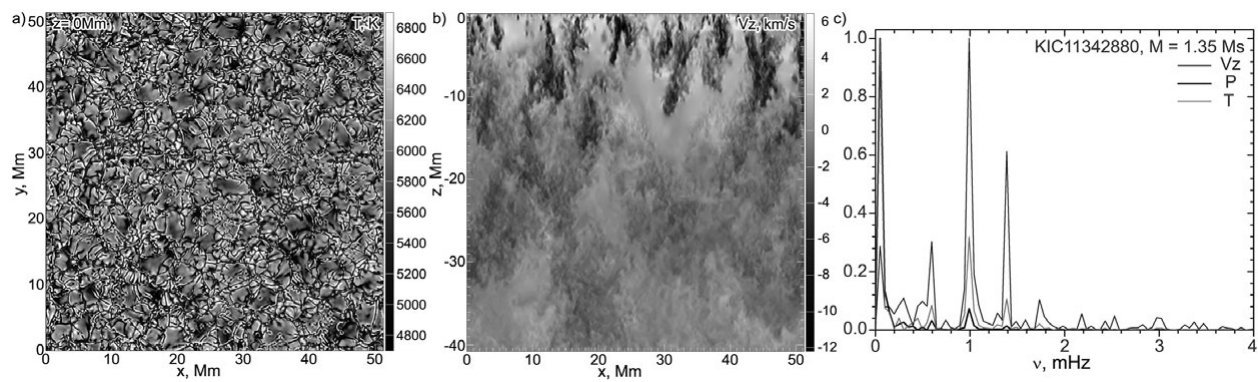

Figure 2. (a) Snapshot of the temperature distribution for the Kepler target star KIC 11342880 $\left(1.35 M_{\odot}, \log T_{\text {eff }}=3.822, \log L / L_{\odot}=0.566\right)$ at the photosphere; $(b)$ vertical cut of the vertical velocity distribution; and $(c)$ the power spectrum of low-degree stellar oscillations for the mean vertical velocity $V_{z}$, pressure $P$, and temperature near the stellar surface.

vertical velocity (panel $b$ ). In the case of KIC $11342880\left(1.35 M_{\odot}\right)$ two basic scales of stellar granulation can be identified: solar-like granulation with a characteristic size of 1-2 Mm, and larger-scale granules, $\sim 3 \mathrm{Mm}$ and larger in size (Fig. 2a). Such doublescale structuring disappears below the photosphere, and in the deeper layers the scale of convective patterns increases. Convective downflows in the intergranular lanes are substantially stronger than the downflows in the solar convection; they can reach up to $16 \mathrm{~km} \mathrm{~s}^{-1}$, and extend up to $40 \mathrm{Mm}$ below the photosphere (Fig. 2b). The realistic numerical simulations make it possible to obtain synthetic oscillation spectra and investigate contributions of various oscillation sources to the observed spectra. This investigation is currently underway. Figure $2 c$ shows an example of the power spectrum for a model of the star KIC 11342880 obtained from the mean vertical velocity, gas pressure and temperature time-series (corresponding to low- $l$ oscillation modes).

Because the stellar convective zone becomes thinner for more massive stars we were able to model the whole convective zone for our second Kepler target KIC $9962653\left(1.47 M_{\odot}\right)$ and investigate the dynamics of the interface between the radiative and convective zones. Figure 3 shows a snapshot of the simulated stellar surface intensity (panel $a$ ), density fluctuations (panel $b$ ) and the vertical velocity (panel $c$ ). In this case, the scale separation between the two populations of small and large granules becomes even stronger, the downdrafts penetrate through the whole convective zone, and overshoot into the radiative zone, reaching speeds up to $30 \mathrm{~km} \mathrm{~s}^{-1}$. Figure $3 b$ shows that in these stars strong downflows overshoot and strongly perturb the upper radiative zone, thus causing excitation of $g$ modes.

\section{Conclusion}

Modern observations of stellar oscillations make it possible to significantly advance understanding of the stellar structure, dynamics and evolution. However, due to the complexity of multi-scale interactions in the highly turbulent stellar convection zones an important step is in building realistic numerical models, where the effects of radiative transfer, ionization, turbulence, internal structure and composition are taken into account from first principles.

We have performed 3D radiative hydrodynamic simulations for a number of selected Kepler target stars (Fig. 1), with masses in the range from 1.01 to $1.52 M_{\odot}$. According to our results, with the increase of stellar mass the convective flows become more and more vigorous, and are characterized by high-speed downflows reaching supersonic velocities (e.g. up to $30 \mathrm{~km} \mathrm{~s}^{-1}$ for $1.47 M_{\odot}$ ) and penetrating into deep convective layers (Fig. 2b). The vigorous convection at the photosphere tends to organize granulation in 

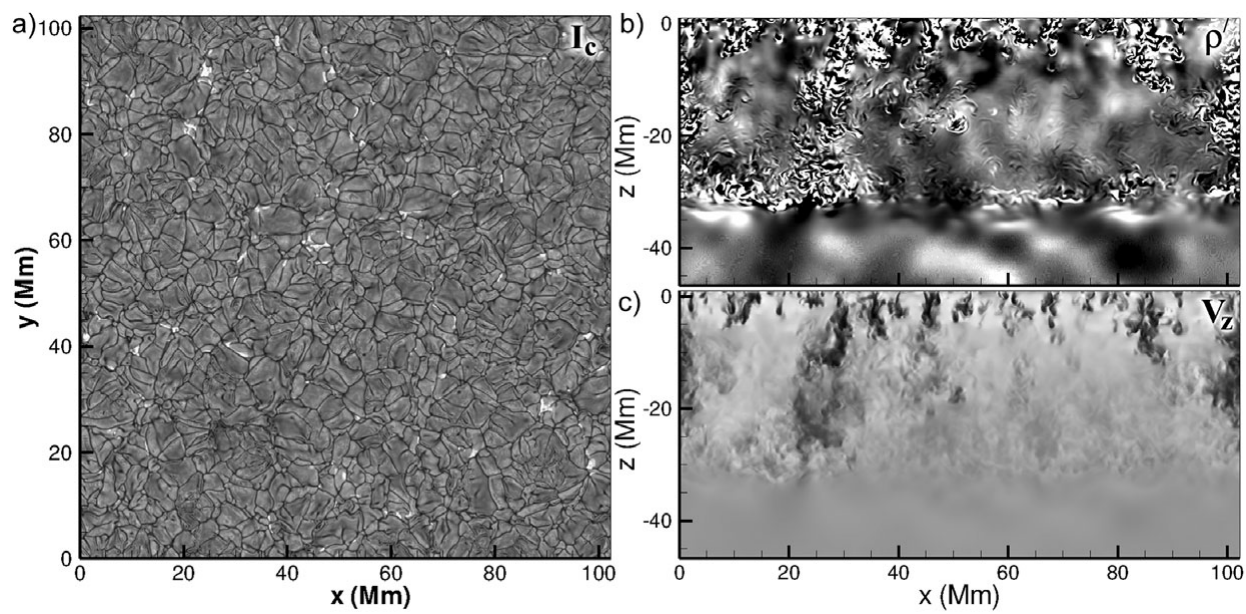

Figure 3. Stellar convection for the Kepler target star KIC 9962653 $\left(M=1.47 M_{\odot}\right.$, $\log T_{\text {eff }}=3.849, \log L / L_{\odot}=0.675$ ): $(a)$ intensity, $(b)$ density fluctuations (vary in range $\pm 2 \cdot 10^{-7} \mathrm{~g} \mathrm{~cm}^{-3}$ ), and $(c)$ vertical velocity (range: -24 to $+10 \mathrm{~km} \mathrm{~s}^{-1}$ ).

two main populations (Figs. 2a, 3a). In more massive F- and A-type stars (examples are KIC 9962653 and KIC 5466537 discussed here) the convective zones become shallow. For these stars we are able to model the whole convective zones including the interface with the radiative zone (Fig. 3 ), which allows us to investigate the mechanism of $g$-mode excitation and characterize the overshooting region.

These preliminary simulations are a first attempt to apply the realistic 'ab-initio' approach, previously used for modeling solar magnetoconvection. This approach allows us to gain insight into the complicated turbulent dynamics of stellar envelopes and use these simulation results for verification and validation of asteroseismology models used for interpretation and analysis of stellar oscillations.

\section{References}

Benomar, O., Baudin, F., Chaplin, W. J., et al. 2012a, MNRAS, 420, 2178

Benomar, O., Bedding, T. R., Stello, D., et al. 2012b, ApJ, 745, L33

Bonaca, A., Tanner, J. D., Basu, S., et al. 2012, ApJ, 755, L12

Chaplin, W. J., Kjeldsen, H., Bedding, T. R., et al. 2011, ApJ, 732, 54

Guzik, J. A. 2011, ApESSS, 336, 95

Guzik, J. A. \& Mussack, K. 2010, ApJ, 713, 1108

Jacoutot, L., Kosovichev, A. G., Wray, A. A., \& Mansour, N. N. 2008a, ApJ, 684, L51

Jacoutot, L., Kosovichev, A. G., Wray, A. A., \& Mansour, N. N. 2008b, ApJ, 682, 1386

Kitiashvili, I. N., Bellot Rubio, L. R., Kosovichev, A. G., et al. 2010, ApJ, 716, L181

Kitiashvili, I. N., Kosovichev, A. G., Mansour, N. N., \& Wray, A. A. 2011a, ApJ, 727, L50

Kitiashvili, I. N., Kosovichev, A. G., Mansour, N. N., \& Wray, A. A. 2011b, Solar Phys., 268, 283

Kitiashvili, I. N., Abramenko, V. I., Goode, P. R., et al. 2013, Physica Scripta, 155, 014025

Kjeldsen, H., Bedding, T. R., \& Christensen-Dalsgaard, J. 2008, ApJ, 683, L175

Lefebvre, S., Kosovichev, A. G., \& Rozelot, J. P. 2006, in: D. Barret, F. Casoli, G. Lagache, A. Lecavelier, \& L. Pagani (eds.), SF2A-2006: Proceedings of the Annual meeting of the French Society of Astronomy and Astrophysics, p. 551

Morel, P. 1997, A\&̊S, 124, 597

Morel, P. \& Lebreton, Y. 2008, Ap\&SSS, 316, 61

Uytterhoeven, K., Moya, A., Grigahcène, A., et al. 2011, A\&A, 534, A125

Verner, G. A., Chaplin, W. J., Basu, S., et al. 2011, ApJ, 738, L28 